\title{
New Non-Target Lesion Identification
}

National Cancer Institute

\section{Source}

National Cancer Institute. New Non-Target Lesion Identification. NCI Thesaurus. Code C139126.

The identification of a non-target tumor, lesion, or site of disease that will be assessed qualitatively over time, and which was not detected at baseline. 